\title{
Embedded Livestock and Poultry Breeding Environment Data Acquisition Method Based on Internet of Things
}

\author{
XU Xiao-hua ${ }^{1}$, CHEN Chang-xi ${ }^{*}$ \\ \{yg69030@163.com ${ }^{1}, x u x h 69850 @ 163$. com $^{2 *}$ \} \\ (1.Tianjin Agricultural University,Tianjin 300384,China; \\ 2.Tianjin Agricultural University, Tianjin 300384,China)
}

\begin{abstract}
The current method of collecting environmental data of livestock and poultry facility breeding cannot automatically screen out abnormal data, so an embedded method of collecting environmental data of livestock and poultry breeding based on Internet of things was designed. First design topology structure of the Internet of things, make between cluster head nodes can communicate with each other, improve the communication ability of member nodes, introducing the environmental perception technology, including the temperature, humidity, ammonia, hydrogen sulfide and carbon dioxide, thermoelectric effect of relying on the thermocouple temperature sensor acquisition, harmful gas depends on the choice of gas molecules light absorption characteristics, absorb certain wavelengths of light conditions to obtain the corresponding information of harmful gas concentration, the final design of acquisition circuit, data acquisition process, completed based on Internet of embedded data acquisition method of design of livestock and poultry breeding environment. The experimental results show that the designed collection method can summarize abnormal data, and separately list the occurrence time and corresponding items of abnormal data, which provides great convenience for controlling the breeding environment.

Key words: Internet of things; Embedded; Livestock and poultry breeding environment; Data acquisition method;
\end{abstract}

\section{Introduction}

China is a large livestock and poultry farming and consumption country. As an important part of agriculture, livestock and poultry farming is of great significance to improving people's diet and living standards, and plays a key role in promoting the rapid development of the national economy. The environment of livestock and poultry breeding is the main factor affecting the growth of livestock and poultry. The environment not only affects the growth of livestock and poultry, but also affects the yield and quality of livestock and poultry [1-2]. With the rise and development of intensive high-density breeding technology, the livestock and 
poultry breeding model has gradually changed from a stocking or low-density low-level artificial breeding model to a scientific, large-scale, mechanized breeding facility breeding model. Although China is a large country in the world for livestock and poultry breeding, the informationization of livestock and poultry facility breeding is still weak. At present, the environmental control of livestock and poultry breeding seriously restricts the improvement of livestock and poultry health and production performance. At present, the data collection methods of livestock and poultry breeding facilities can not automatically screen out abnormal data, so an embedded data collection method of livestock and poultry breeding environment based on the Internet of things is designed. First, design the topology of the Internet of Things so that the cluster head nodes can communicate with each other, improve the communication capabilities of member nodes, and introduce environmental sensing technologies, including the sensing of temperature, humidity, ammonia, hydrogen sulfide, and carbon dioxide. The temperature depends on the thermoelectric effect of thermocouple sensor to collect, and the harmful gas depends on the selective absorption characteristics of gas molecules to detect the absorption of a certain wavelength of light to obtain the corresponding concentration information of harmful gas. Finally, a collection circuit is designed to implement the data collection process.

\section{Research on Embedded Data Collection Method of Livestock and Poultry Based on Internet of Things}

The overall design of the embedded livestock and poultry breeding environment data collection method adopts design concepts such as low power consumption and general equipment ideas. Multi-sensors are added to the breeding environment, and the interaction between the sensors is completed through the Internet of Things. The overall structure of aquaculture environment data collection is shown in the figure: 


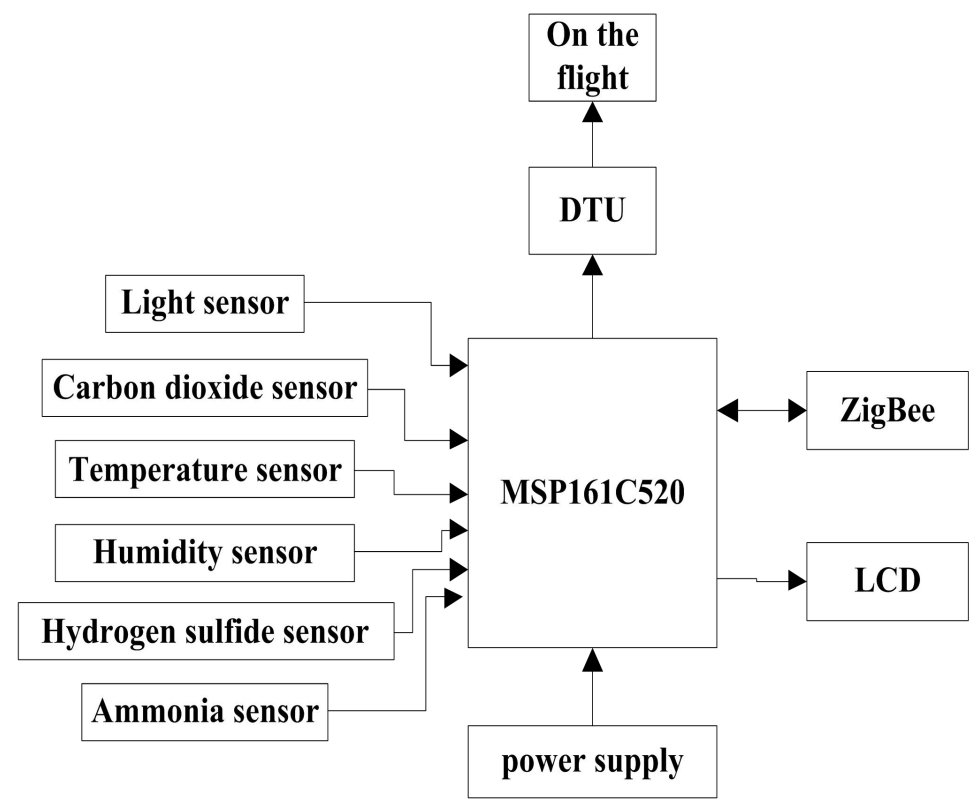

Figure 1 Overall structure of data collection for breeding environment

As can be seen from the above figure, the overall structure of the breeding environment data collection consists of a power supply, a microprocessor, a ZigBee communication module, a temperature and humidity sensor, an illumination sensor, a carbon dioxide sensor, a hydrogen sulfide sensor, and an ammonia sensor. After the composition, the real-time information of the environmental parameters is displayed on the LCD display[3-4]. Next, a comprehensive study was conducted on the collection methods of livestock and poultry environmental data.

\subsection{Designing the topology of the Internet of Things}

The topology of the IoT network refers to the networking structure of ZigBee nodes, which directly affects the network's working performance. Therefore, it is necessary to select a specific ZigBee network topology for different applications and functional characteristics. The existing ZigBee network topology can be divided into flat network, hierarchical network, hybrid network and Mesh network according to the functions of the nodes [5]. This article mainly uses ZigBee's hierarchical network as the field network structure for breeding environment data collection, as shown in the following figure: 


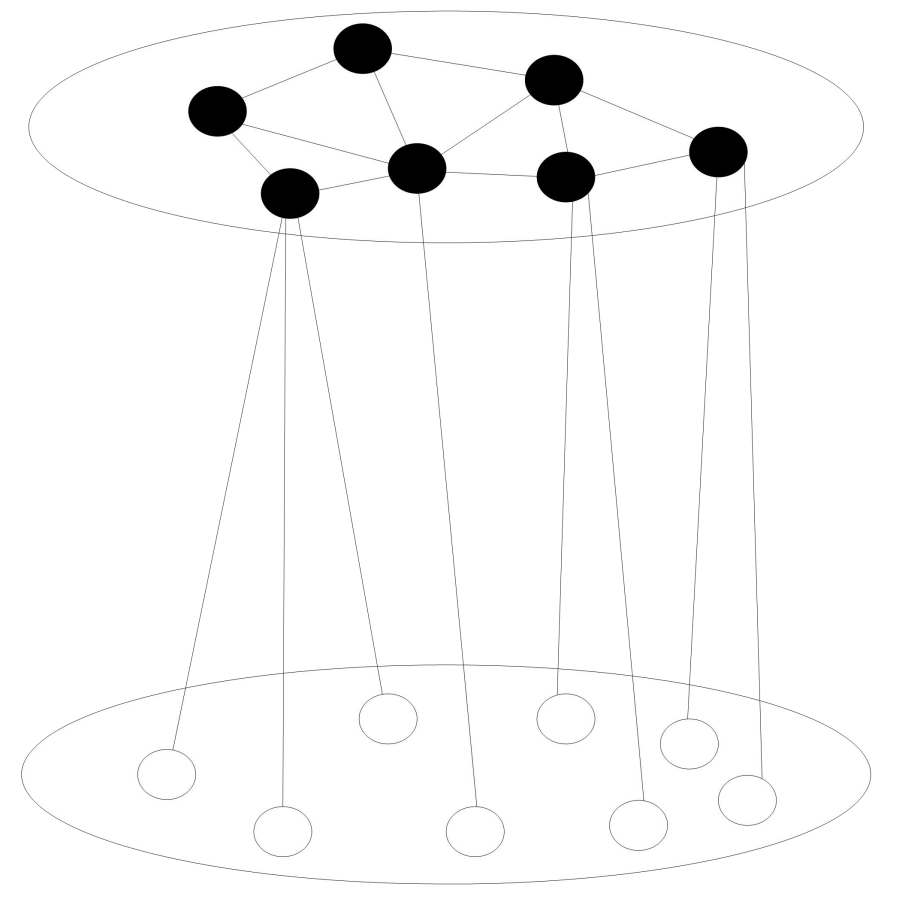

Figure 2 ZigBee's hierarchical network structure

Hierarchical network structure is an extension of the traditional flat network structure, and the peer network nodes are divided into cluster head nodes with data aggregation and network organization capabilities and member nodes with data aggregation and forwarding functions only. In a hierarchical network, only the cluster head nodes have perfect layer and network layer protocols, which determines that only the cluster head nodes can communicate with each other, and the member nodes can only collect data and communicate with their cluster head nodes. Hierarchical network improves the routing structure and management efficiency, but increases the cost of hardware [6]. Another disadvantage that cannot be ignored is the poor communication ability of the member nodes, which reduces the robustness of the network. Based on the above analysis, the data transmission from the sensing data collection site to the remote data center is completed in the transmission mode of ZigBee and GPRS relay. The designed topology is shown in the following figure: 


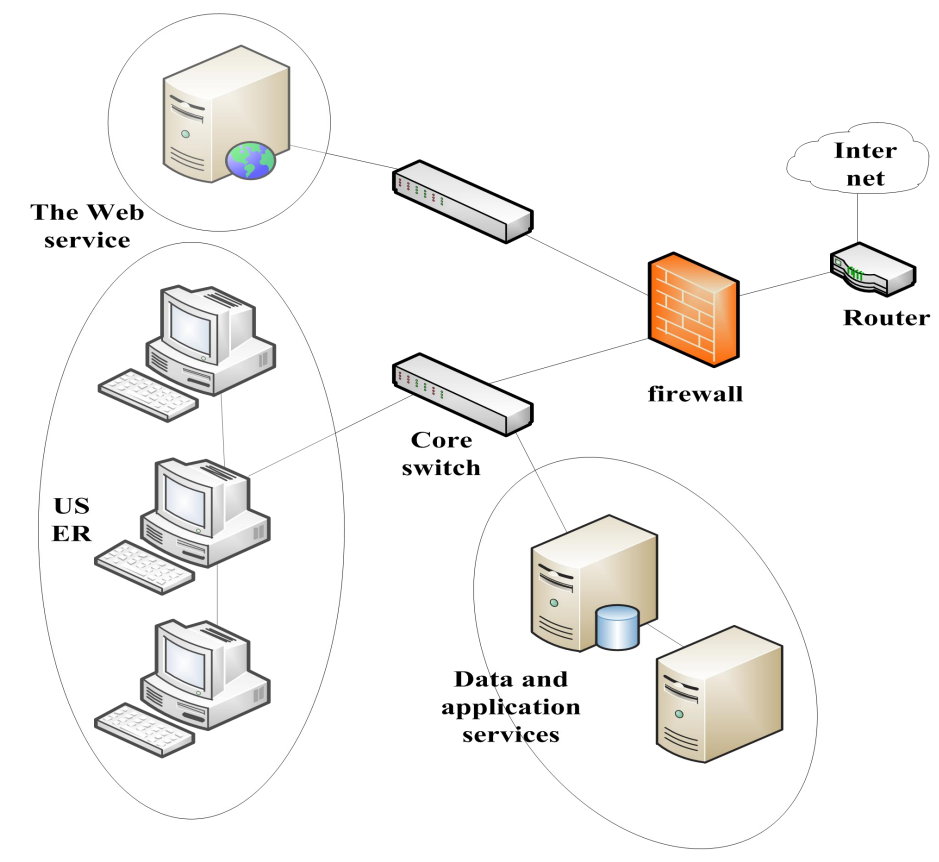

Figure 3 Topological structure of aquaculture environment data collection method

The topology consists of sensor nodes deployed on pallets, turnover boxes, and ZigBee network coordinators installed in storage vehicles and warehouses. The coordinator and the GPRS data transmission unit are designed to connect hardware. Through the server and the intranet application server. The data is released as content to the public and intranet users.

\subsection{Introduction of environmental awareness technology}

Livestock and poultry facilities breeding environment perception technology is a composite technology that integrates multi-disciplines such as electronic technology, opto-electromechanical technology, computer and information processing, and is comprehensively used. It is widely used in data collection for livestock and poultry breeding environment. The sensitivity and stability of the environmental monitoring sensors for livestock and poultry facilities breeding performance directly determine the quality of the environmental data collected from livestock and poultry [7]. Reliable and accurate environmental perception is the basis for subsequent data collection analysis and processing. Temperature sensing is used to detect the cold and hot degree of the livestock and poultry breeding environment. In actual livestock and poultry breeding environment temperature sensing applications, most of them use negative temperature coefficient thermistor sensors: 


$$
R_{t}=R_{0} e^{B\left(\frac{1}{T}-\frac{1}{T_{0}}\right)}
$$

In the above formula, $T$ represents the measured temperature, the unit is $\mathrm{K} ; T_{0}$ in $T=273+t$ represents the reference temperature, the unit is $\mathrm{K} ; T_{0}=273+t_{0}, R_{t}$ and $R_{0}$ represent the resistance of the thermistor at the temperature of $T, T_{0}$, and $B$ is the thermistor Material constant. The value of $B$ can be calculated:

$$
B=\frac{\ln \left(\frac{R_{t}}{R_{0}}\right)}{\left(\frac{1}{T}-\frac{1}{T_{0}}\right)} \text { (2) }
$$

According to the definition of the temperature coefficient of the thermistor, the relative change in resistance $\alpha$ can be expressed as:

$$
\alpha=\frac{1}{R_{t}} \frac{d R_{t}}{d t}=-\frac{B}{T^{2}}
$$

Without the need for an external power supply, the thermistor uses a thermocouple temperature sensor to convert the temperature conversion into a potential change sensor. The thermoelectric effect of the thermocouple sensor is shown in the figure:

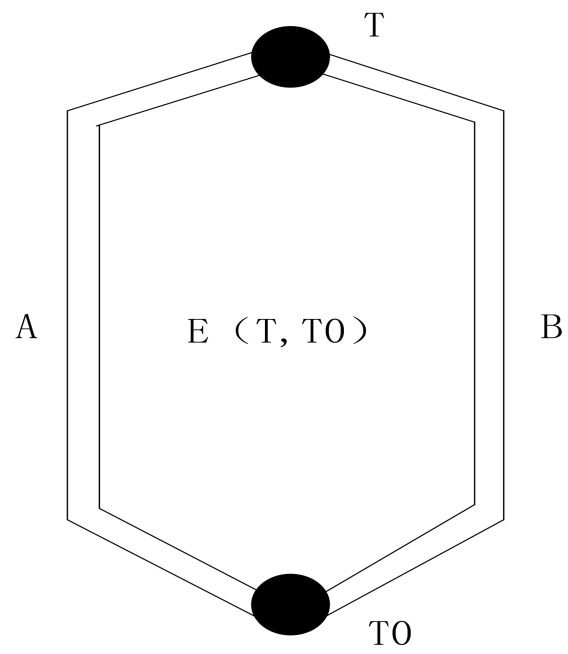


Figure 4 Thermoelectric effect of thermocouple sensor

In the closed loop shown in the figure above, there are two different metals A and B. If the temperature of metal A and metal B is different, that is, $T>T_{0}$, then a thermoelectric potential $E\left(T, T_{0}\right)$ will be generated in this closed loop. By detecting the potential, Capable of sensing temperature changes and completing temperature collection.

Another important aspect of environmental perception is the perception of harmful gases. It is necessary to use carbon dioxide sensors, hydrogen sulfide sensors, and ammonia sensors to perform real-time online monitoring of harmful gases in livestock and poultry facilities. At present, electrochemical gas sensors in China are characterized by high measurement accuracy, fast speed, easy use, good environmental applicability, and low price [8]. There are many applications for sensing harmful gases in livestock and poultry facilities. The performance analysis of the infrared carbon dioxide sensor is shown in the following table:

Table 1 Performance analysis of electrochemical and infrared carbon dioxide sensors

\begin{tabular}{ccc}
$\begin{array}{c}\text { Performance } \\
\text { indicators }\end{array}$ & Electric chemical formula & Infrared type \\
\hline Sensitivity & Low & High \\
Stability & low & High \\
Volume & Large & Small \\
Anti-interference & weak & Strong \\
ability & Low & slightly higher \\
Cost & Short & Long \\
Service life & Large & Small \\
Post maintenance &
\end{tabular}

It can be seen from the above table that the basic detection principle of harmful gases is the selective absorption of light by gas molecules, that is, gas molecules can only absorb photons whose energy is exactly equal to the difference between two energies. When a beam of infrared monochromatic light or composite light passes through the gas to be measured, if the molecule of the gas to be measured selectively absorbs the light in some frequency band of the radiation light, the absorption spectrum will be generated; According to the gas molecular structure, the gas absorption spectrum of different molecular structure is different from each other. By detecting the absorption of a certain wavelength of light, the concentration information of the corresponding harmful gas can be obtained qualitatively and quantitatively.

\subsection{Design data acquisition circuit}


The data acquisition circuit designed in this paper is mainly completed by TD SIM4 100, and its specific circuit is shown in the figure below:

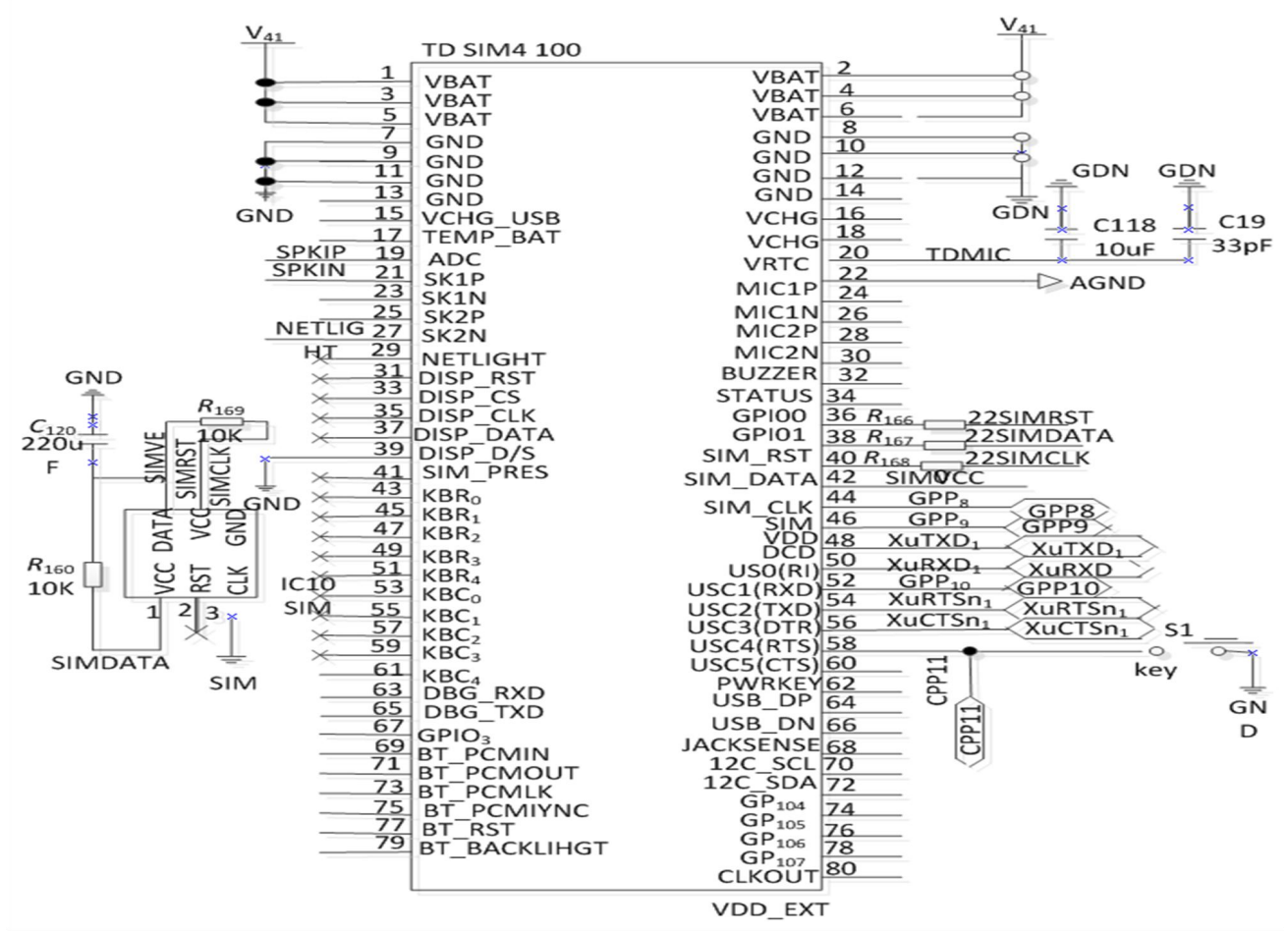

Figure 5 Interface circuit of TD SIM4 100

TD SIM4 100 is a multi range, 12 bit DAC chip, its working voltage is only $5 \mathrm{~V}$. During operation, the circuit can receive analog signals higher than the power supply voltage and lower than the ground potential; the chip has 8 independent analog input channels; four programmable input ranges are provided for the input analog signals: $\pm 10 \mathrm{~V}, \pm 5 \mathrm{~V}, 0-5 \mathrm{~V}$, $0-10 \mathrm{~V}$, which increase the effective dynamic input range to 14 bits [9]. The chip provides a flexible interface for 4-20 mA signal and sensor powered by $\pm 12 \mathrm{~V}$ or $\pm 15 \mathrm{~V}$ to single $5 \mathrm{~V}$ system; the chip has $5 \mathrm{MHz}$ bandwidth, $100 \mathrm{ksps}$ throughput; internal / external clock and internal / external start selection, $8+4$ parallel data interface, internal $4.096 \mathrm{~V}$ or external reference voltage; it has low current shutdown mode controlled by software and hardware. Among them, the input and output of 8-bit tristate data I / O port are compatible with TTL or CMOS logic level. Its data conversion process starts from writing the control word, and after the completion of data conversion of TD SIM4 100, a standard notification signal will be given. The system can control its configuration to be query mode or interrupt mode to realize 
data reading. See the following table for specific control conditions:

Table 2 Analog input range control

\begin{tabular}{ccc}
\hline BIP & RNG & Analog input range/V \\
\hline 0 & 0 & $0-5$ \\
0 & 1 & $0-10$ \\
1 & 0 & \pm 5 \\
1 & 1 & \pm 10 \\
\hline
\end{tabular}

Firstly, the initialization of sc2410 system resources is carried out in the system initialization program of S3C2410, including the I / O mode, the DMA controller of interrupt system, etc. then, the initialization process of GPRS module is completed, and the UART0 of S3C2410 is configured as FIFO mode through the work mode configuration. Then, according to the GPRS communication protocol, use the serial port to send at instruction to initialize GPRS, connect the remote data service, and establish the data path. After the above initialization process is completed, start the data collection process [10-12]. The specific process of collection is shown in the figure below:

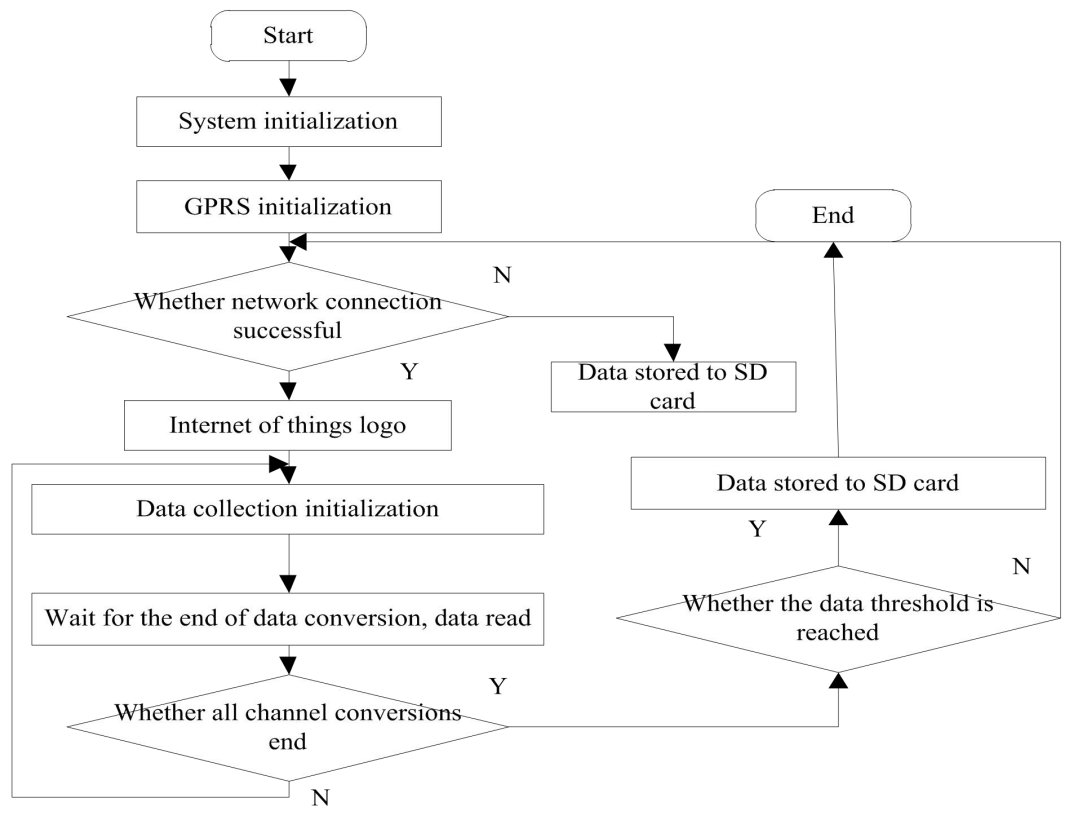

Figure 6 Data collection process

In the process of data acquisition, different analog input range and sampling mode are selected according to the characteristics of different channel signals by programming. At the same time, the conversion is started. After the conversion, the 12 bit data is read into memory twice. Finally, when all the 8-way data are converted, the data sending program is called, and 
the sampling data is sent to the Internet of things terminal through GPRS module for data processing. So far, the research on the data collection method of embedded livestock and poultry breeding environment based on the Internet of things has been completed.

\section{Experiment}

In order to verify the effectiveness of this method, we choose a pig house for experimental test. We use the traditional data collection method and the data collection method designed in this paper to collect the environmental data of the pig house at the same time, and compare the collection results.

\subsection{Experiment preparation}

The tested pigsty is located to the south, with a length of $100 \mathrm{~m}$, a width of $15 \mathrm{~m}$, a spacing of $35 \mathrm{~m}$, bilateral fences, and about 20 domestic pigs in the shed. There are windows on the north and south walls of the pigsty, and two doors on the East and west sides, which play the role of ventilation and lighting, and three sensor nodes are installed in them, as shown in the following figure:

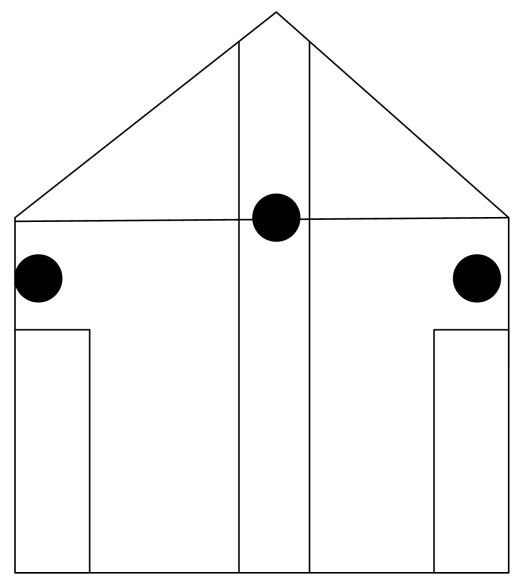

Figure 7 Pig house sensing node placement

The outdoor temperature is about $25{ }^{\circ} \mathrm{C}$, as shown in the figure above, three sensor nodes are installed in the livestock house, including two bit routing nodes and one coordinator node. The black dot in the figure represents the position of the sensor. Node 1 is installed on the right side of the livestock house door, with a height of $1.5 \mathrm{~m}$ from the ground. Node 2 is installed on the wall in the middle of the livestock house, with a height of $2 \mathrm{~m}$ from the ground. Node 3 is installed on the south side of the livestock house. The height of the wall from the ground is $1.5 \mathrm{~m}$. The test period of the system is 15 days, and the time interval of data upload is 10 minutes. The server analyzes the data according to the data uploaded by the central node, and then obtains the real-time environment parameters. 


\subsection{Experimental results and analysis}

According to the above experimental process, the embedded livestock and poultry breeding environment data collection results of this method and the embedded livestock and poultry breeding environment data collection results of the traditional method are respectively obtained, as shown in the following figure:

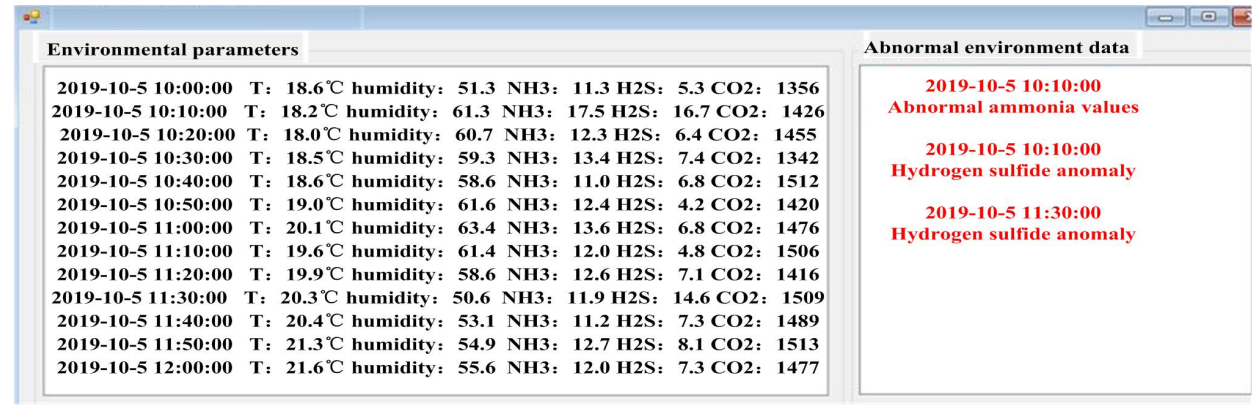

Figure 8 Data acquisition results of this method

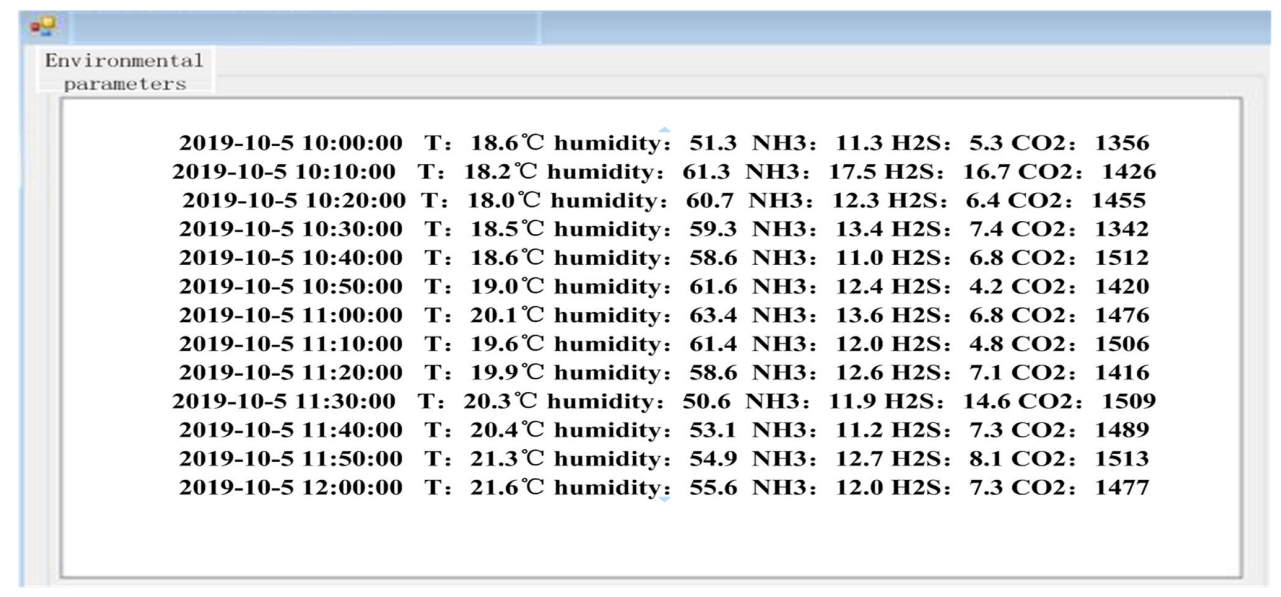

Figure 9 Acquisition results of traditional methods

It can be seen from the comparison of the acquisition results of the above two methods that the traditional acquisition method and the acquisition method designed in this paper can collect the temperature, humidity, ammonia value, hydrogen sulfide value and carbon dioxide value in the pig house. But for the abnormal values beyond the normal range, the traditional methods can not be directly reflected; and the collection method designed in this paper can summarize the abnormal data, list the time of abnormal data and the corresponding items separately, which provides great convenience for controlling the breeding environment.

In order to further verify the effectiveness of the method in this paper, the accuracy of data collection of embedded livestock and poultry breeding environment of the traditional 
method and the method in this paper are compared and analyzed, and the comparison results are shown in Figure 10.

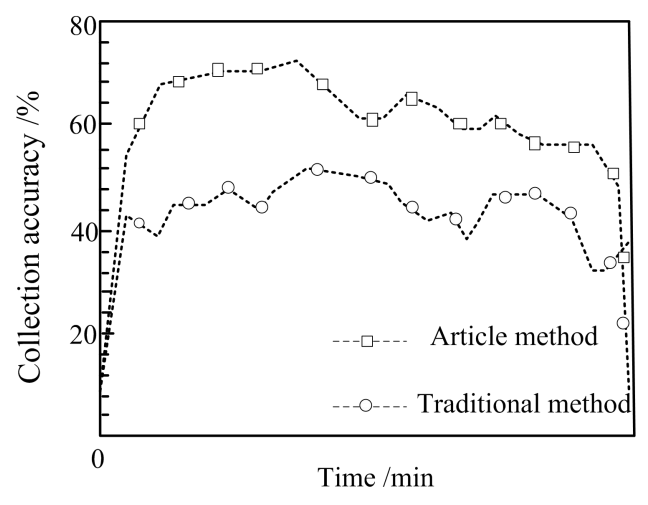

Figure 10 comparison results of acquisition accuracy

According to the analysis of Figure 10, the accuracy rate of the embedded livestock and poultry breeding environment data collection of this method is higher than that of the traditional method.

\section{Concluding}

In order to better control the breeding environment of livestock and poultry and have an intuitive understanding of the abnormal data generated by the breeding environment, an embedded data collection method based on the Internet of things is designed. Firstly, the topological structure of the Internet of things is designed, and the environmental sensing technology is introduced, including the perception of temperature, humidity, ammonia, hydrogen sulfide and carbon dioxide. Finally, the collection circuit is designed to realize the data collection process. The experimental results show that the designed collection method can summarize the abnormal data, and enumerate the occurrence time and corresponding items of the abnormal data separately, which provides great convenience for controlling the breeding environment. In the research of the embedded livestock and poultry breeding environment data collection method based on the Internet of things, because the embedded livestock and poultry breeding environment monitoring is not in place, there are still some deficiencies in the collected embedded livestock and poultry breeding environment data. In the future, we will focus on the embedded livestock and poultry breeding environment monitoring to improve the monitoring accuracy, so as to ensure the integrity of the embedded livestock and poultry breeding environment data Sex.

\section{Fund projects}

Post Scientist of National Broiler Industry Technology System（CARS-41-G25） 
The Science and Technology Planning Program of Tianjin Science and Technology Bureau, China (18ZXRHNC00080)

Information Post of Tianjin Dairy Industry Technology System Innovation Team(ITTCRS2020023)

\section{Reference}

[1] Ling, R. Xujun, Z. Zaotong, Z. et al.: Environment Monitoring System of Livestock and Poultry House Based on Internet of Things and MCGS Software. Techniques of Automation and Applications, Vol. 37 no. 08, pp. 50-52 (2018)

[2] Tao, C. Yun, W. Xinyan, Z. et al.: A massive sample data acquisition method for intelligent classification of remote sensing images. Bulletin of Surveying and Mapping, no. 10, pp. 56-60 (2019)

[3] Zhuoran, L.: Design of Aquaculture Network Monitoring System Based on Embedded Linux. Journal of Agricultural Mechanization Research. Vol. 41 no. 11, pp. 229-233 (2019)

[4] Shengtao, Z. Qing, C. Hui, L. et al.: Analysis on application of Internet of Tings in industrial aquaculture. Fishery Modernization, Vol. 46 no. 04, pp. 8-13 (2019)

[5] Peixian, L. Changan, W. Xuechun, Y. et al.: An Environmental Monitoring System for a Mirror Carp Culture Pond Based on Internet of Things. Chinese Journal of Fisheries, Vol. 32 no. 02, pp. $49-54$ (2019)

[6] Juan, H. Fan, W. Weijian, C. et al.: Development of water quality monitoring system of aquaculture ponds based on narrow band internet of things. Transactions of the Chinese Society of Agricultural Engineering, Vol. 35 no. 08, pp. 252-261 (2019)

[7] Xuefeng, X. Yuanyuan, L.: Design and Implementation of aquaculture Monitoring System Based on Internet of Things. Modern Informationn Technology, Vol. 2 no. 11, pp. 175-178+182 (2018)

[8] Yuqing, L. Jiajia, L. Shouqi, C. et al.: Design and application of monitoring system for crab breeding base based on internet of things. Transactions of the Chinese Society of Agricultural Engineering, Vol. 34 no. 16, pp. 205-213 (2018)

[9] Minghua, S. Yuanjie, M. Cuijie, L. et al.: Design and Implementation of Lighting Intensity Collector for Marine Shellfish Culture IoT Platform. Shandong Agricultural Sciences, Vol. 50 no. 11, pp. 154-158 (2018)

[10] Min, L. Guohuan, W. Shi, Y.: Simulation Optimization of Data Acquisition Efficiency for Multi-Port Communication Information Transmission Computer Simulation, Vol. 35 no. 03, pp. 115-118 (2018)

[11] Shakoor, N. Lee, S. Mockler Todd, C.: High throughput phenotyping to accelerate crop breeding and monitoring of diseases in the field. Current Opinion in Plant Biology, Vol. 38, pp. 184-192 (2017)

[12] Kaiying, W. Xiaoyang, Z. Yong H.: Review on noninvasive monitoring technology of poultry behavior and physiological information. Transactions of the Chinese Society of Agricultural Engineering, Vol. 33 no. 20, pp. 197-209 (2017) 